\title{
Relación entre diabetes mellitus y enfermedad periodontal
}

\author{
NAVARRO SANCHEZ AB * \\ FARIA ALMEIDA $\mathbf{R}$ * \\ BASCONES MARTINEZ $\boldsymbol{A}$ * *
}

Navarro Sánchez AB, Faria Almeida R, Bascones Martínez A. Relación entre diabetes mellitus y enfermedad periodontal. Av Periodon Implantol. 2002; 14, 1: 9-19.

\begin{abstract}
RESUIMEN
La asociación entre diabetes mellitus y la enfermedad periodontal ha sido motivo de estudio durante mucho tiempo. Son varias las hipótesis que se barajan a la hora de explicar dicha relación. El propósito de este artículo es revisar los estudios publicados en la literatura periodontal hasta la fecha.
\end{abstract}

\section{PALABRAS CLAVE}

Diabetes mellitus, hiperglucemia, periodontitis

\section{INTRODUCCIÓN}

La diabetes mellitus (DM) es la enfermedad endocrina más frecuente e incluye un grupo de trastornos metabólicos caracterizados por la elevación de los niveles de glucosa en sangre acompañados de complicaciones a largo plazo.

Puede ser clasificada en dos categorías principales: > Diabetes mellitus insulino-dependiente o tipo I.

- Diabetes mellitus no-insulino dependiente o tipo II.

La diabetes mellitus tipo I se debe a la destrucción probablemente de etiología autoinmune, de las células beta de los islotes del páncreas dando como resultado niveles plasmáticos de insulina bajos o indetectables. El inicio es normalmente antes de los 40 años de edad, puede ser agudo, con sed, poliuria, polifagia y pérdida de peso. La enfermedad se controla mediante inyecciones diarias de insulina y es característicamente inestable en episodios de cetoacidosis.

La diabetes mellitus tipo II es de inicio insidioso, apareciendo en individuos de edad media como resultado de una utilización defectuosa de la insulina, siendo los niveles plasmáticos de insulina en valores absolutos, normales o altos. Estos pacientes no presentan episodios de cetoacidosis y controlan la hiper- glucemia mediante dieta y/o hipoglucemiantes orales. Un elevado porcentaje de estos pacientes presenta problemas de obesidad.

\section{ASPECTOS EPIDEIMIOLÓGICOS}

La asociación entre diabetes mellitus y la enfermedad periodontal ha sido motivo de estudio durante mucho tiempo.

Clásicamente se ha atribuido una relación directa entre la diabetes mellitus y la incidencia y severidad de la enfermedad periodontal, sin embargo, los diversos estudios publicados en la literatura muestran resultados aparentemente contradictorios.

Existen 5 razones que podrían explicar estas discrepancias:

1. La existencia de 2 tipos clínicos de DM bien diferenciados, los cuales han sido, a menudo, indistintamente tratados en los estudios.

2. Los grupos control se han considerado como "no diabéticos" simplemente por determinación de glucemia basal, sin tener en cuenta medidas analíticas más precisas.

3. Se han utilizado diferentes índices de destrucción periodontal.

* Alumno Máster de Periodoncia.

** Catedrático de Medicina Bucal y Periodoncia. Facultad de Odontología (Universidad Complutense de Madrid). 
4. Las variables se han estudiado aplicando distintos métodos estadísticos.

5. Los grupos de población estudiados han sido pequeños para permitir realizar cortes de edad de suficiente entidad para ser significativo.

Numerosos estudios epidemiológicos, tanto transversales como longitudinales, han encontrado una gran prevalencia de periodontitis en pacientes diabéticos comparados con controles sanos. A continuación se revisará la literatura al respecto publicada en esta última década para minimizar las diferencias metodológicas existentes entre los muchos estudios que se han publicado a lo largo de los años. Nos limitaremos a los últimos diez años ya que la terminología y criterios utilizados están relativamente estandarizados.

Los indios Pima presentan la mayor incidencia conocida de DM tipo II del mundo; sobre el $40 \%$ de los mayores de 35 años presentan DM tipo II. Un estudio clásico señala una alta prevalencia y severidad de la periodontitis en esta población. Sin embargo, tenemos que tener presente que estos datos van referidos a esta población específica por lo que se cuestiona su generabilidad o validez externa.

Estudios que fueron realizados con pacientes diabéticos insulino-dependientes o tipo I mostraron resultados similares a los encontrados para los diabéticos no insulino-dependientes.

En ambos tipos de pacientes diabéticos no parece existir ninguna relación entre la prevalencia o la severidad de la periodontitis y la duración de la diabetes.

Los pacientes diabéticos bien controlados, evaluados según sus niveles de hemoglobina glicosilada en sangre, presentan menor severidad de la periodontitis comparados con los mal controlados. Por lo tanto, los mayores esfuerzos terapéuticos deberían ir dirigidos a prevenir la aparición de periodontitis en aquellos sujetos con riesgo de desarrollar diabetes mellitus.

Estudios recientemente publicados sugieren que si existe un control efectivo de la periodontitis en pacientes diabéticos los niveles en suero de los productos finales de glicosilación avanzados se reducen, y por tanto, se mejora el estado de la diabetes. Si esto se confirmara en estudios futuros, el control de la infección peridontal debería ser considerada como parte integral del control diabético.

\section{ESTADO DE LA PERIODONTITIS EN DIABÉTICOS}

De los cinco estudios epidemiológicos publicados sobre diabéticos tipo I, tres de ellos tienen un diseño transversal (1-3) y dos son longitudinales (4-5), y aunque existen marcadas diferencias en los protocolos, en todos ellos se pudieron observar diferencias significativas en relación a las profundidades de sondaje, pérdida ósea evidenciada radiográficamente y pérdida de inserción clínica. Los resultados de dichos estudios indican que los diabéticos insulino-dependientes presentan mayor prevalencia de periodontitis y mayor severidad ya que las profundidades de sondaje, la pérdida ósea y la pérdida de inserción clínica resultó mayor que en los controles. En los estudios longitudinales además se pudo observar que los diabéticos mal controlados presentaban una periodontitis más severa en comparación a los que estaban bien controlados.

De los cinco estudios epidemiológicos publicados sobre diabéticos tipo II, cuatro de ellos tienen un diseño transversal (6-9) y uno es longitudinal (10), y aunque principalmente se limitaron a una población específica, los indios Pima, donde se ha observado una gran prevalencia de este tipo de diabetes, los resultados de dichos estudios indican que los diabéticos tipo II presentan una mayor prevalencia de periodontitis que los controles. Así la incidencia de periodontitis en los indios Pima es 2.6 veces mayor en los diabéticos que en los no diabéticos. En los estudios longitudinales también se pudo observar que los diabéticos mal controlados presentan una periodontitis más severa frente a los que estaban bien controlados.

\section{RESULTADOS COIMPARATIVOS DEL TRATAMIENTO PERIODONTAL EN DIABÉTICOS Y NO DIABÉTICOS}

Existen dos estudios que han evaluado la respuesta al tratamiento periodontal en pacientes diabéticos y no diabéticos $(11,12)$. El primero tuvo una duración de 34 meses de seguimiento tras la aplicación de tratamiento periodontal no quirúrgico y el segundo con una duración de 5 años evaluó tanto tratamiento quirúrgico como no quirúrgico. Ambos estudios incluyeron en sus muestras tanto sujetos tipo I como tipo II, y aunque existen diferencias en los protocolos, los autores llegaron a las mismas conclusiones. Los resultados indican que no existen diferencias en la respuesta al tratamiento periodontal entre pacientes diabéticos y no diabéticos, sin embargo, debemos tener en cuenta que el tamaño de muestra fue demasiado pequeño.

\section{EL EFECTO DE UN BUEN CONTROL METABÓLICO DE LA DIABETES SOBRE LA PERIODONTITIS}

El único estudio publicado al respecto es el que realizaron Satrowijoto et al (13) con una duración de 8 
meses en el cual seleccionaron 6 pacientes tipo I con una duración de su diabetes $\geq 5$ años y trataron de realizar un control metabólico de la diabetes mediante la administración repetida de insulina y un programa de educación. Los pacientes durante el período que duró el estudio no recibieron tratamiento periodontal. Los resultados indican una marcada reducción de los niveles en sangre de $\mathrm{HbA}_{1 \mathrm{c}}$ (indica el nivel del control metabólico de los 3 meses previos), mientras que en el baseline el valor medio fue de $11.2 \%$, a los 4 meses fue de $9.1 \%$ y de $9.6 \%$ a los 8 meses. Se observó mejoría en los signos de inflamación gingival, sin embargo no fue así para las medidas de periodontitis.

Los resultados de distintos estudios epidemiológicos han mostrado periodontitis más severa en aquellos diabéticos mal controlados, sugiriendo que el control de la diabetes podría afectar a la presentación clínica de periodontitis, pero para sacar conclusiones definitivas se deben realizar estudios que establezcan una relación causa-efecto (14).

\section{EL EFECTO DEL TRATAMIENTO PERIODONTAL SOBRE EL CONTROL METABÓLICO DE LA DIABETES}

Aunque numerosos autores han estudiado la relación entre la presencia de diabetes, el nivel de control metabólico y el riesgo de presentar periodontitis y el grado de severidad de la misma, pocos son los estudios que han evaluado esta misma relación pero a la inversa, es decir, el efecto de la periodontitis sobre el nivel de control metabólico. ¿La presencia o severidad de la periodontitis afecta el control metabólico del paciente diabético?, ¿puede el tratamiento periodontal tener un efecto medible sobre dicho control?

Taylor et al (15) realizaron en sujetos diabéticos tipo 2 exámenes clínicos y radiográficos para determinar si la periodontitis severa aumentaba el riesgo de presentar un pobre control metabólico. Todos los sujetos presentaron niveles de $\mathrm{HbA}_{1 \mathrm{c}}<9 \%$ en el baseline (buen control metabólico), fueron reevaluados a los 2 y 4 años. Se observó que una gran proporción de sujetos con periodontitis severa preentaba un pobre control metabólico $\left(\mathrm{HbA}_{1 \mathrm{c}}>9 \%\right)$ durante el seguimiento. Los resultados del estudio mostraron clara asociación entre la presencia de periodontitis severa y riesgo incrementado de mal control metabólico.

Hasta la fecha son cuatro los estudios que han evaluado el papel del tratamiento periodontal sobre el control metabólico de la diabetes:

1.- En 1960, Williams \& Mahan (16) realizaron extracciones y terapia quirúrgica sobre 9 pacientes diabéticos. Registraron los parámetros de control glucémico de forma periódica definido como los niveles de glucosa en sangre y la dosis diaria de insulina. Observaron que 7 de los 9 pacientes presentaron mejoría en su estado periodontal y que en éstos se redujeron las necesidades de insulina incluso en $>50 \%$.

2.- En 1992, Miller et al (17) evaluaron el efecto de la terapia no quirúrgica más doxiciclina sistémica durante 14 días sobre el control glucémico de 9 diabéticos tipo $1 \mathrm{mal}$ controlados con periodontitis. Las reevaluaciones post-tratamiento se realizaron a las $4 \mathrm{y}$ 8 semanas. Se observó que 5 de los 9 pacientes presentaron mejoría significativa en el sangrado al sondaje, lo que se vio acompañado de una reducción significativa de los niveles de $\mathrm{HbA}_{1 \mathrm{c}}$, y por tanto, mejoría del control metabólico. Igualmente se pudo observar que los 4 sujetos restantes que no presentaron mejoría en el sangrado en el sondaje tampoco lo hicieron en sus niveles de $\mathrm{HbA}_{11}$. Este estudio carece de controles pero sugiere que una mejoría del estado periodontal puede verse acompañado de forma paralela de una mejoría en el control metabólico de pacientes diabéticos mal controlados.

3.- En 1995, Aldridge et al (18), por el contrario, en un ensayo clínico controlado randomizado realizado sobre 12 pacientes diabéticos tipo 1 moderadamente bien controlados, que presentaban periodontitis severa y que recibieron terapia no quirúrgica y 10 pacientes diabéticos tipo 1 controles que no recibieron terapia, observaron a las 6 semanas mejoría del estado periodontal, en el grupo que recibió tratamiento periodontal pero no encontraron diferencias en relación a la mejoría del control metabólico, en el grupo control no se observó mejoría ni en el estado periodontal ni en el control metabólico.

La $H_{b} A_{I C}$ nos proporciona una valoración del control glucémico >6-8 semanas previas a la toma, por tanto, como el seguimiento se hizo a las 6 semanas esta prueba de la $\mathrm{HbA}_{i c}$ no valoró con precisión los posibles cambios acontecidos.

4.- En 1996, Grossi et al (19) examinaron 85 diabéticos tipo 2 mal controlados con periodontitis a los 3,6 y 12 meses tras recibir terapia periodontal mecánica en combinación con doxiciclina sistémica $100 \mathrm{mg} /$ día o un placebo durante 14 días. El grupo que recibió doxiciclina presentó mayor reducción de la profundidad de sondaje, mayor ganancia de inserción clínica y presentaron una reducción significativa de los niveles de $\mathrm{HbA}_{1 \mathrm{c}}$ a los 3 meses, sin embargo, posteriormente los niveles de $\mathrm{HbA}_{1 \mathrm{c}}$ volvieron a los niveles iniciales en baseline e incluso se incrementaron. El grupo que recibió el placebo no presentó mejoría en los niveles de $\mathrm{HbA}_{1 \mathrm{c}}$. Los resutlados del estudio indican que la terapia mecánica combinada con doxiciclina sistémi- 
ca no sólo mejora el estado periodontal de diabéticos tipo 2 mal controlados sino que a corto plazo también mejora su control glucémico.

Tras revisar estos cuatro estudios, parece que la terapia periodontal en pacientes diabéticos no va a ir asociada necesariamente con una mejoría del control metabólico si previamente al mismo los diabéticos presentaban un buen control glucémico, pero sí podría resultar en una mejoría en aquellos diabéticos mal controlados.

¿Cuál es el mecanismo mediante el cual la hiperglucemia afecta al periodonto o cómo podría la periodontitis modular el control metabólico? Los productos finales de glicosilación avanzados (AGEs) se acumulan en presencia de una hiperglucemia prolongada y su formación altera la función de numerosos componentes de la matriz extracelular modificando las interacciones matriz-matriz y matriz-células. Estas alteraciones tienen un efecto adverso sobre los tejidos diana, especialmente sobre la estabilidad del colágeno y la integridad vascular (20). Los monocitos, macrófagos y células endoteliales poseen receptores de alta afinidad para estos AGEs. La unión de AGEs al receptor del macrófago va a dar lugar a una secreción aumentada de IL-1, TNF- $\alpha$ y factor de crecimiento insulie-like, mientras que la unión de AGEs al receptor de la célula endotelial va a dar lugar a cambios en la coagulación que conllevan una trombosis focal y vasoconstricción. Se sabe que estos eventos mediados por AGEs tienen una gran importancia en la patogénesis de las complicaciones de la diabetes, como la retinopatía, neuropatía, nefropatía y aterosclerosis, pero puede que también estén implicados en la patogénesis de la periodontitis incrementando la destrucción del tejido a este nivel.

Sabemos que el control glucémico afecta la resistencia del huésped frente a la infección, sin embargo el mecanismo por el cual un pobre control metabólico aumenta la susceptibilidad a las infecciones permanece aún desconocido. Las investigaciones hasta la fecha han mostrado reducción en la quimiotaxis, fagocitosis y muerte intracelular de neutrófilos en diabéticos mal controlados, sin embargo no se conoce si esto es clínicamente suficiente para predisponer a estos pacientes a las infecciones.

También se sabe que la presencia de infecciones produce una resistencia de los tejidos frente a la insulina y agrava el control metabólico, y que esto sucede tanto en sujetos diabéticos como en no diabéticos. Además se sabe que el aumento de la resistencia tisular frente a la insulina persiste durante un período de tiempo extenso una vez desaparecida la infección.

Por todo esto se plantea la hipótesis de que la infec- ción periodontal, puesto que es una infección bacteriana crónica, podría igualmente aumentar la resistencia tisular frente a la insulina y conllevar un mal control glucémico. Como nuestro tratamiento periodontal tiene como objetivo controlar dicha agresión bacteriana y reducir la inflamación, podría restaurar esta situación tisular con el tiempo, resultando en una mejoría del control metabólico del diabético. Estudios a largo plazo que observaron una mejoría en el control glucémico de diabéticos tras recibir terapia periodontal apoyan esta hipóteiss, otros estudios a corto plazo no han observado los mismos resultados posiblemente porque no han permitido que pase el suficiente tiempo para que se restaure la situación tisular de resistencia frente a la insulina o bien porque no han resuelto la infección.

\section{LA PERIODONTITIS Y LA DIABETES MELLITUS: RELACIÓN BIDIRECCIONAL}

Existe gran evidencia en la literatura periodontal sobre la influencia que los factores del huésped ejercen sobre la patogénesis y la progresión de la periodontitis. Offenbacher revisó cómo los distintos mecanismos del huésped afectan y modifican la expresión de esta enfermedad (21).

Se han presentado modelos de patogénesis en los cuales los desórdenes sistémicos que afectan la función de los neutrófilos, monocitos y/o linfocitos alterando la producción o actividad de citoquinas y otros mediadores de la inflamación, conllevan frente a la agresión bacteriana un incremento localizado o generalizado de pérdida de inserción.

Se ha documentado muy bien el impacto que diversos desórdenes sistémicos tienen sobre el periodonto, sin embargo, todavía permanecen sin aclarar las consecuencias que las periodontitis conllevan sobre el estado de salud general.

Las investigaciones más actuales en el campo de la "Perio-Medicina", término acuñado por Offenbacher (World Workshop 1996), sugieren que existe una relación mucho más compleja de la que se ha podido apreciar previamente y los resultados de diversos estudios indican la necesidad de una cooperación más estrecha entre médicos y odontólogos con el fin de establecer un enfoque integral para el manejo de la salud de estos pacientes.

Se presenta el modelo patogénico por el cual la periodontitis aumenta la severidad de la diabetes mellitus al complicar el control metabólico y vicever- 
sa. Según este modelo, la combinación de dos vías distintas explicaría la mayor destrucción tisular observada en pacientes diabéticos periodontales, y cómo la periodontitis complica la severidad de la diabetes y el grado de control metabólico, estableciendo que la relación entre periodontitis y diabetes mellitus sea bidireccional.

\section{LA PERIODONTITIS Y LA DIABETES MELLITUS:}

Si revisamos todos los estudios epidemiológicos publicados, existe suficiente evidencia científica para establecer que la diabetes mellitus supone un factor de riesgo para desarrollar periodontitis (21) y que la periodontitis es una complicación de la diabetes, de hecho, se la conoce como la sexta complicación de la diabetes mellitus (22).

Además existe también evidencia científica para establecer que la severidad de la periodontitis se incrementa con una mayor duración de la diabetes (23). Igualmente la mayor duración de la diabetes se relaciona con una mayor prevalencia y severidad de otras complicaciones propias de la diabetes como son la retinopatía, la nefropatía, la neuropatía, la microangipatía y macroangiopatía (24).

\section{LAS INFECCIONES Y LA DIABETES MELLITUS:}

Las infecciones alteran el estado metabólico-endocrinológico del huésped dificultando el control de sus niveles de azúcares en sangre (25).

Las infecciones bacterianas producen resistencia de los tejidos frente a la insulina al estimular la secreción de citoquinas, fundamentalmente TNF- $\alpha$ e IL-1 que disminuyen la acción de la misma sobre los tejidos (26).

El receptor para la insulina tirosina kinasa, la expresión de segundos mensajeros y la acción de la proteína kinasa $\mathrm{C}$, de forma individual o en conjunto, median algunos de los efectos de la insulina, como son la traslocación y activación de las proteínas transportadoras de glucosa (27). El estudio de Kanety (28) sugiere que el TNF- $\alpha$ es el principal responsable de inducir resistencia tisular frente a la insulina al suprimir la fosforilación del receptor para la insulina IRS-1 (insulin receptor substrate-1).

Las infecciones por tanto conllevan resistencia de los tejidos frente a la insulina y mal control de la diabetes al favorecer el estado de hiperglucemia, aumentándose así el riesgo de aparición de complicaciones diabéticas (29).

\section{LA PERIODONTITIS}

Las periodontitis son infecciones bacterianas crónicas producidas por bacterias principalmente anaerobias gramnegativas que colonizan el surco gingival y se fijan a las superficies dentarias. Para que una especie bacteriana pueda ser considerada como un patógeno periodontal tiene que disponer de dos capacidades principales, por un lado poder colonizar el área subgingival y por otro producir factores que, o bien dañen directamente los tejidos del huésped, o bien induzcan a que estos tejidos se destruyan (30).

Las reacciones del huésped tienen una función importante en la patogénesis de las periodontitis al contribuir al proceso patológico o modular los efectos de las bacterias. Son varios los componentes que participan en la respuesta inmune: neutrófilos, macrófagos, linfocitos, células plasmáticas, complemento, anticuerpos, citoquinas... pudiendo afectar la colonización por las bacterias, la invasión bacteriana y la destrucción hística puesto que las reacciones inmunes pueden ser beneficiosas (papel protector) o resultar nocivas para el huésped cuando dichas respuestas son exageradas al desencadenar destrucción tisular.

La periodontitis afecta a los tejidos de soporte de los dientes causando pérdida de inserción por destrucción del tejido conectivo y reabsorción del hueso alveolar $(31,32)$, pero también conllevan una serie de cambios a nivel sistémico sobre las células inmunocompetentes y células que participan en la respuesta inflamatoria $(33,34)$.

Offenbacher realizó una exhaustiva revisión sobre la patogénesis de la periodontitis y el papel de las bacterias y los mediadores de la inflamación responsable de la destrucción tisular. Las bacterias periodontopatógenas al presentar una serie de factores de virulencia que, o bien son secretados, o bien forman parte de la estructura del microorganismo (lipopolisacáridos, ácidos grasos de cadena corta, toxinas...) interaccionan con los mecanismos inmunes del huésped desencadenando la liberación de mediadores de la inflamación con carácter catabólico, principalmente IL-1 $\beta$, IL$6, \mathrm{PGE}_{2}$ y TNF- $\alpha$ causando destrucción del tejido conectivo y reabsorción ósea.

\section{LA HIPERGLUCEMIA Y LAS COMPLICACIONES DE LA DIABETES MELLITUS}

Una exposición prolongada de hiperglucemia es el principal factor responsable para desarrollar complicaciones en el paciente diabético como la retinopatía, nefropatía y neuropatía $(35,36)$.

La hiperglucemia da lugar a la formación de produc- 
tos finales de glicosilación avanzados (AGEs). Los AGEs son compuestos derivados de la glicosilación de proteínas y lípidos de forma no enzimática y de carácter irreversible que se acumulan en el plasma, paredes de los vasos sanguíneos y los tejidos. Son los principales responsables de causar expansión de la matriz extracelular tras su unión al colágeno, causando el endurecimiento y engrosamiento de las paredes de los vasos sanguíneos. El engrosamiento de las paredes de los vasos sanguíneos es el resultado de la combinación por un lado de la reducción en la degradación de los AGEs y por otro del aumento en la propia síntesis de los componentes de dicha matriz. Esta proliferación de la matriz extracelular es debida principalmente a la producción local aumentada de factores de crecimiento como TNF- $\alpha$, IL-1 y PDGF.

Los macrófagos presentan receptores de alta afinidad para los AGEs (RAGE) y su unión determinará la puesta en marcha de la síntesis de perfiles citoquínicos, principalmente se liberarán TNF- $\alpha$ e IL-1. Estas citoquinas tienen la capacidad de unirse a varias estirpes celulares que participan en la remodelación tisular en condiciones normales. Sin embargo, la excesiva acumulación de AGEs por la situación de hiperglucemia desencadenará la degradación del tejido conectivo.

También se ha demostrado que los AGEs se acumulan en el tejido gingival de los pacientes diabéticos. Los resultados del estudio de Schmidt et al (37) demuestran que en pacientes adultos diagnosticados de diabetes que fueron sometidos a tratamiento periodontal quirúrgico, el tejido gingival presentaba mayores cantidades de AGEs que en los pacientes no diabéticos. Los autores plantean como hipótesis que la interacción entre los AGEs y los RAGE presentes en las células endoteliales y los fagocitos mononucleares a nivel del tejido gingival va a conllevar una sobreestimulación de las mismas que conducirá a una respuesta inflamatoria crónica.

\section{RELACIÓN BIDIRECCIONAL ENTRE LA PERIODONTITIS Y LA DIABETES MELLITUS}

La mayor parte de destrucción del tejido conectivo que tiene lugar en la periodontitis es debida a la interacción de las bacterias periodontopatógenas y sus productos con las células inmunocompetentes y los fibroblastos al provocar la activación y secreción local de mediadores de la inflamación con carácter catabólico, principalmente IL-1 $\beta, \mathrm{PGE}_{2}$, TNF- $\alpha$ e IL-6 (38).

El mecanismo biológico aceptado en la actualidad que intenta explicar el porqué de que los pacientes diabéticos presenten periodontitis más severas defiende, por un lado que la acumulación de AGEs afectaría la migración y la fagocitosis tanto de poli- morfonucleares como de mononucleares, resultando en el establecimiento de una flora subgingival que por maduración se irá transformando en predominantemente anaerobia gramnegativa. Esto va a desencadenar la secreción de mediadores solubles que mediarán la destrucción del tejido conectivo y la reabsorción ósea, y el establecimiento de un estado de resistencia de los tejidos frente a la insulina. Simultáneamente por otro lado, la infección periodontal también inducirá a un estado de resistencia de los tejidos frente a la insulina contribuyendo a la hiperglucemia y consecuentemente a la acumulación de AGEs.

La relación que se establece entre diabetes mellitus y periodontitis, por tanto es bidireccional resultando en una mayor severidad de la periodontitis y una mayor dificultad para controlar los niveles de glucosa en sangre en pacientes diabéticos.

\section{PATOGÉNESIS DE LA PERIODONTITIS EN PACIENTES DIABÉTICOS}

La diabetes mellitus es un grupo heterogéneo de desórdenes con diferentes causas, pero todos ellos caracterizados por hiperglucemia, una deficiencia relativa o absoluta de insulina o resistencia frente a la misma y la tendencia de desarrollar ciertas complicaciones a largo plazo. Las complicaciones más comunes incluyen la aterosclerosis, la retinopatía diabética, la nefropatía diabética y la neuropatía diabética.

Las complicaciones de la diabetes mellitus se deben a cambios estructurales y funcionales que aparecen en tejidos susceptibles. Son varios los mecanismos que se han planteado como responsables: alteración del metabolismo del mioinositol, la glicosilación no enzimática, alteraciones hemodinámicas y factores genéticos. Sin embargo, en la actualidad se desconoce todavía cuál o cuáles son los responsables directos de la patogénesis de dichas complicaciones, al igual que tampoco se ha determinado si son diferentes los mecanismos involucrados según el tejido afectado.

Nishimura et al $(39,39)$ presentan un estudio realizado sobre una muestra de 24 adolescentes japoneses con diabetes mellitus tipo I planteando la hipótesis que tanto los episodios de hiperglucemia como los de hipoglucemia podrían contribuir en la patogénesis de la periodontitis en este tipo de pacientes diabéticos, ya que los diabéticos tipo I a menudo presentan ambos tipos de episodios. Con este propósito realizan un examen periodontal de los sujetos que fueron seleccionados para el estudio y presentan una serie de datos de laboratorio para determinar los factores 
de riesgo en relación a las condiciones diabéticas y muestran también una serie de datos biológicos in vitro que resultan del estudio de las células del ligamento periodontal cultivadas a distintos niveles de glucosa.

Según sus observaciones sugieren que la hiperglucemia podría, de forma indirecta, exacerbar la destrucción del tejido periodontal partiendo de la base que el estado de hiperglucemia va a inducir la glicosilación progresiva de diversas proteínas del organismos dando lugar a los llamados productos finales de glicosilación avanzados (AGEs) que actuarán sobre los fagocitos como factores quimiotácticos y a los cuales se van a unir vía receptores específicos RAGE, MSR quedando así activados para que liberen radicales de oxígeno y niveles exagerados de citoquimas proinflamatorias como la IL-1, IL-2 y TNF- $\alpha$, que van a exacerbar la respuesta inflamatoria y por tanto contribuirán a una mayor destrucción tisular a nivel periodontal, y que tanto la hiperglucemia como la hipoglucemia podrían de forma directa alterar las funciones biológicas de dicho tejido a través de interacciones células-matriz.

\section{LOS PACIENTES DIABÉTICOS TIENEN MAYOR RIESGO DE PERIODONTITIS}

Hasta la fecha, son varias las hipótesis que se barajan a la hora de explicar el mayor riesgo que presentan los pacientes diabéticos para presentar periodontitis, entre ellas cambios vasculares, alteración en el metabolismo del colágeno y disfunción de los polimorfonucleares neutrófilos (alteración en la quimiotaxis y la fagocitosis). Entre otros, los estudios realizados por Monouchehr-Pour et al (40) y Bissada et al (41) demostraron además que esta disfunción de los neutrófilos era mayor en aquellos diabéticos mal controlados.

Los resultados del examen clínico muestran que de los 24 adolescentes japoneses diabéticos tipo I que fueron seleccionados para este estudio (edades comprendidas entre los 7 y los 24 años), 17 presentaban signos de inflamación gingival y de estos 17, 3 de ellos presentaban pérdida de inserción evidenciada clínica y radiográficamente, siendo por ello diagnosticados de periodontitis. Además 4 pacientes presentaban alguna de las complicaciones diabéticas clásicas particularmente la retinopatía, 1 de ellos también presentaba periodontitis y 2 gingivitis. La mayoría de los pacientes que presentaron alguna complicación incluyendo la periodontitis, tenían una evolución de su diabetes de al menos 12 años. Esto podría indicarnos que la duración de la diabetes podría ser un importante factor en el desarrollo de complicaciones diabéticas. Curiosamente el control metabólico no se relacionó con el inicio de las complicaciones.
Los resultados del laboratorio que valoraron las funciones de neutrófilos y linfocitos no mostraron diferencias significativas, ni en la quimiotaxis, ni en la fagocitosis, ni tampoco en la actividad bacteriana. Tampoco se observó alteraciones ni en el recuento linfocitario, ni en sus funciones. Sin embargo, sí observaron que de los 3 sujetos diabéticos que presentaron periodontitis, 2 de ellos presentaban niveles elevados de citoquinas proinflamatorias en el suero, al igual que otro sujeto que presentaba retinopatía, pero no periodontitis. Los autores sugieren que estos niveles incrementados de citoquinas proinflamatorias podrían deberse a niveles incrementados de AGEs, que desencadenarían esa liberación de citoquinas exagerada. Sin embargo, no pudieron evidenciar los niveles incrementados de AGEs utilizando anticuerpos específicos.

\section{NIVELES ELEVADOSY BAJOS DE GLUCOSA MODULAN LAS FUNCIONES BIOLÓGICAS DE LAS CÉLULAS DEL LIGAMENTO PERIODONTAL IN VITRO VIA INTEGRINAS}

Se sabe que los diabéticos presentan alterada su capacidad de cicatrización. La hiperglucemia provoca un aumento en la producción de proteínas de matriz extracelular como la fibronectina, laminina, colágeno tipo IV... que además de conllevar un engrosamiento de la membrana basal de los vasos sanguíneos (causando microangiopatía) van a intervenir modulando funciones celulares. Así, por ejemplo, la hiperglucemia causa la diferenciación de los macrófagos para que adquieran un fenotipo catabólico causando daño tisular en lugar de anabólico que sería el responsable de liberar factores de crecimiento como el factor derivado de las plaquetas, factor de crecimiento fibroblástico... que inducen la reorganización tisular. Ambas situaciones conllevarán la alteración del ratio células-matriz en los tejidos.

Las interacciones células-matriz son mediadas por unos receptores de superficie celular específicos conocidos como integrinas para las correspondientes proteínas de matriz. El exceso de proteínas de matriz extracelular en los tejidos causa una alteración directa de las funciones celulares vía moléculas de integrinas. Nishimura et al (42) informaron que los fibroblastos del ligamento periodontal en humanos cultivados in vitro a altas concentraciones de glucosa (450 $\mathrm{mg} / \mathrm{dL}$ ) presentaban una mayor expresión del receptor para la fibronectina (VLA-5) que las mismas células cultivadas a concentraciones normales fisiológicamente. Cultivadas a bajas concentraciones (10 mg/dL) mostraban una menor expresión de dicho receptor y muerte celular. Por tanto, las concentraciones de glucosa regulan la expresión de VLA-5 en dichas células. Sabemos que el incremento en los niveles de recep- 
tores para la fibronectina causa la alteración directa de funciones celulares de los fibroblastos como por ejemplo su proliferación, migración y adhesividad. Así los fibroblastos del ligamento periodontal en humanos cultivados in vitro a altas concentraciones de glucosa expresaron mayores niveles de VLA-5 y mostraron una mayor adhesividad y menor respuesta quimiotáctica cuando se los estimulaba con factor de crecimiento derivado de las plaquetas que en un importante quimioatrayente para dichas células.

El mecanismo de regulación del VLA-5 vía variaciones en los niveles de glucosa permanece aún desconocido.

\section{FACTORES QUE POTENCIALMENTE PODRÍAN CONTRIBUIR AL DESARROLLO DE PERIODONTITIS EN PACIENTES DIABÉTICOS}

Aunque una gran cantidad de factores contribuye a la pérdida de inserción en pacientes diabéticos (flora bacteriana, alteración en la respuesta del huésped...), se sabe que la presencia de hiperglucemia, característica común a las distintas formas de diabetes, es un factor predisponente determinante en la patogénesis de la periodontitis en el paciente diabético.

\section{FUNCIÓN DE LOS LEUCOCITOS POLIMORFONUCLEARES}

En los pacientes diabéticos se ha observado que la función de los polimorfonucleares (PMN) está disminuida. Esta alteración en su función afecta a la quimiotaxis, la adherencia y la fagocitosis alterando la respuesta del huésped frente a la infección $(43,44)$.

Manouchhr-Pour et al (45) observaron que los diabéticos con periodontitis severa presentaban una menor quimiotaxis de los PMN en comparación con diabéticos con periodontitis leve o sujetos no diabéticos con periodontitis leve o severa.

McMullen (46) et al plantearon en un estudio llevado a cabo sobre los miembros de una misma familia con historia de diabetes y periodontitis severa que esta reducción de la quimiotaxis de los PMN podría tener un origen genético.

Bissada et al (47) observaron que la actividad fagocítica de los neutrófilos en el surco gingival era menor que la de los neutrófilos de sangre periférica y, que independientemente del estado metabólico del diabético, la actividad funcional de los PMN aislados de las localizaciones enfermas era menor que en los de las localizaciones sanas.

Golub et al (48) realizaron un estudio sobre ratas a las que químicamente indujeron diabetes y observaron, que tras la aplicación de agentes quimiotácticos (FMLPs y caseína) colocados de forma atraumática sobre el margen gingival, la respuesta quimiotáctica de los neutrófilos fue menor en las ratas diabéticas que en las ratas controles, y que las ratas diabéticas a las que se les estaba administrando insulina presentaban una mejor respuesta, sugiriendo que dichas alteraciones en los PMN podrían ser corregidas mediante terapia con insulina.

Cutler et al (49) en un informe realizado sobre un paciente diabético tipo I atribuyeron la alteración en la fagocitosis, quimiotaxis y lisis de los PMN frente a un periodontopatógeno aislado, la Porphyromonas gingivalis, a una inhibición de la vía glucolítica del PMN, un metabolismo anormal de los nucleótidos cíclicos y una reducción en los receptores de membrana de los PMN.

\section{LASS INFECCIONES EN PACIENTES DIABBÉTICOS}

Generalmente se acepta que los diabéticos son más susceptibles a desarrollar infecciones que los sujetos no diabéticos. También se cree que las infecciones en los diabéticos son más raras que en los no diabéticos. Sin embargo, en la actualidad no existen estudios que aporten conclusiones firmes y apoyen estas impresiones clínicas.

Existen estudios que han demostrado que la respuesta del huésped está alterada en los diabéticos, específicamente la función de los neutrófilos está disminuida.

También se ha demostrado que en presencia de infecciones, los tejidos presentan una resistencia a la acción de la insulina, pero las bases moleculares para comprender esto no están claras todavía.

Existen estudios que han demostrado cambios vasculares en el periodonto de sujetos diabéticos caracterizados por un engrosamiento de la membrana basal de los vasos capilares gingivales. Se piensa que estas alteraciones impiden la difusión de oxígeno, la migración de PMN y la difusión de factores séricos como los anticuerpos.

Posiblemente las alteraciones en la función de los PMN, la resistencia de los tejidos a la acción de la insulina y los cambios vasculares contribuyan al tiempo para que el diabético sea más susceptible a las infecciones en general y a la periodontitis en particular. Parece ser que si se controlan los niveles de glucosa en suero estas alteraciones se pueden revertir en gran medida y por tanto se podrían controlar dichas infecciones. 


\section{CICATRIZACIÓN DE LAS HERIDAS EN PACIENTES DIABÉTICOS:}

La cicatrización de las heridas en los pacientes diabéticos está comprometida, sin embargo los mecanismos responsables permanecen aún desconocidos. Probablemente la alteración de la actividad de distintas estirpes celulares no sólo juegue un papel primordial en la mayor susceptibilidad que estos pacientes presenta frente a las infecciones sino que también afecte la respuesta de cicatrización.

Niveles elevados de glucosa parecen afectar la síntesis, maduración y homeostasis del colágeno.

Willershauschen-Zonchen et al (50) realizaron un estudio sobre fibroblastos humanos de piel y demostraron que en condiciones de hiperglucemia se produce una reducción en la proliferación y crecimiento de los fibroblastos y una reducción en la síntesis de colágeno y glicosaminoglicanos. Se ha demostrado igualmente que los fibroblastos de la encía y del ligamento periodontal en sujetos diabéticos también sintetizan menos colágeno que en los sujetos no diabéticos.

Además de una menor producción de colágeno en diabéticos también se ha observado que presentan una mayor actividad colagenasa en el fluido crevicular gingival. Se piensa que la principal fuente de esta colagenasa son los neutrófilos más que las bacterias. Sorsa et al (51) demostraron que estos niveles incrementados de colagenasa podían ser inhibidos in vitro por tetraciclinas.

El presencia de hiperglucemia tiene lugar un proceso de glicosilación no enzimática y oxidación de proteínas (incluida el colágeno) y lípidos para reducir los azúcares. En primer lugar se forman unos productos con carácter reversible conocidos como bases Schiff y productos Amadori (52). Un ejemplo de este tipo de productos es la $\mathrm{HbA}_{\mathrm{lc}}$, utilizada en la clínica como marcados de control de la glucosa a largo plazo en pacientes diabéticos. Posteriormente tienen lugar una serie de reacciones moleculares complejas que dan como resultado la formación de los llamados productos finales de glicosilación avanzados (AGEs). Los AGEs pertenecen a un grupo heterogéneo de compuestos de carácter irreversible, a este grupo pertenecen la carboximetilisina, la piralina y la pentosidina. Se ha visto que la forma de AGEs más predominante en pacientes diabéticos y relacionada con el envejecimiento tisular es la carboximetilisina. Los AGEs no sólo se acumulan durante el envejecimiento y en aquellas situaciones de hiperglucemia sino también en aquellos estados caracterizados por un acúmulo incrementado de lipoproteínas modificadas y el retraso del aclaramiento de proteínas como sucede en la amiloidosis.

Se sabe que los AGEs juegan un papel central en la patogénesis de las complicaciones del paciente diabético y se han descrito una gran cantidad de receptores celulares para los AGEs. El que mejor ha sido caracterizado es el RAGE perteneciente a la superfamilia de las inmunoglobulinas de moléculas de superficie celular (53). El RAGE está presente sobre la superficie de ciertas células diana en diabéticos, como por ejemplo en células endoteliales, en fagocitos mononucleares y ciertas células del sistema nervioso central y periférico. De la interacción entre los AGEs y los RAGE tiene lugar la apuesta en marcha de mecanismos de señalización intracelular que conllevan una alteración en el fenotipo celular. Esta alteración propicia la generación de un ambiente proinflamatoria resultando en el desarrollo de lesiones e los vasos y una alteración de la respuesta reparativa normal.

De la integración entre los AGEs y el RAGE presente en la superficie celular de macrófagos resultará la liberación de mediadores con carácter proinflamatorio con IL-1, IL-6 y TNF- $\alpha$ que a su vez activarán a los osteoclastos y a las metaloproteínas conllevando reabsorción ósea y destrucción del conectivo.

De la integración entre los AGEs y el RAGE presente en la superficie celular de las células endoteliales resultará la mayor expresión de la selectina VCAM-1 (molécula de adhesión) favoreciendo la perpetuación de la respuesta inflamatoria y cambios que conllevan una trombosis focal y vasoconstricción.

El estudio de Schneir et al (54) demostró que la glicosilación del colágeno preexistente en los márgenes de la herida disminuye su solubilidad alterando su remodelación y además por otro lado la mayor actividad colagenasa existente en el diabético degrada el nuevo colágeno sintetizado retrasando y dificultando todo ello la cicatrización de la herida.

\section{SUMMARY}

The association between diabetes mellitus und Periodontal. Disease have been studied for a long time. There are several hypothesis which have tried to explain this relation. The purpose of this paper is to review the Periodontal literature up to day

\section{KEY WORDS}

Diabetes mellitus, hiperglycemia, periodontitis

\section{BIBLIOGRAFÍA}

1. De Pommereau V, Dargent-Pare C, Robert JJ, Brian M. 
Periodontal status in insulin-dependent diabetic adolescents. J Clin Periodontol 1992; 19: 628-632

2. Safkan-Seppälä B, Ainamo J. Periodontal conditions in insulin-dependent diabetes mellitus. J Clin Periodontol 1992; 19: 24-29

3. Thorstensson H, Hugoson A. Periodontal disease experience in adult long-duration insulin-dependent diabetes. J Clin Periodontol 1993; 20: 352-358

4. Seppälä B, Seppälä M, Ainamo J. A longitudinal study on insulin-dependent diabetes mellitus and periodontal disease. J Clin Periodontol 1993; 20: 161-165

5. Firatli E. The relation between clinical periodontal status and insulin-dependent diabetes mellitus. J Periodontol 1997; 68: 136-140

6. Nelson RG, Shlossman M, Budding LM, et al. Periodontal disease and NIDDM in Pima Indians. Diabetes Care 1990; 13: 836-840

7. Shlossman M, Knowler WC, Pettit DJ, Genco RJ. Type 2 diabetes mellitus and periodontal disease. J Am Dent Assoc 1990; 121: 532-536

8. Emrich LJ, Shlossman M, Genco RJ. Periodontal disease in non-insulin dependent diabetes mellitus. J Periodontol 1992; 62: 123-130

9. Unal T, Firatli E, Sivas A, Meric $\mathrm{H}, \mathrm{Oz} \mathrm{H}$. Fructosamine as a possible monitoring parameter in non-insulin dependent diabetes mellitus patients with periodontal disease. J Periodontol 1993; 64: 191-194

10. Taylor GW, Burt BA, Becker MP, et al. Severe periodontitis andrisk for poor glycemic control in patients with non-insulin dependent diabetes mellitus. J Periodontol 196; 67: 1085-1093

11. Pervonen T, Knuuttila M, Pohjamo L, Nurkkala H. Immediate response to non-surgical periodontal treatment in subjects with diabetes mellitus. J Clin Periodontol 1991; 18: 65-68

12. Westfelt E, Rylander H, Blohme G, Jonasson P, Lindhe J. The effect of periodontal therapy in diabetics. Results after 5 years. J Clin Periodontol 1996; 23: 92-100

13. Sastrowijoto SH, van der Velden U, van Sttenbergen TJM, et al. Improved metabolic control, clinical periodontal status and subgingival microbiology in insulin-dependent diabetes mellitus. J Clin Periodontol 1990; 17: 235242

14. Mealey BL. Influence of periodontal infections on systemic health. Periodontology 2000. 1999; 21: 197-209
15. Taylor GW, Burt BA, Becker MP, Genco RJ, Sholssman M, Knowler WC, Pettitt DJ. Severe periodontitis and risk for poor glycemic control in patients with non-insulin dependent diabetes mellitus. J Periodontol 1996; 67: 1085-1093

16. Williams RC, Mahan CJ. Periodontal disease and diabetes in young adults. JAMA 1960; 172: 776-778

17. Miller LS, Manwell MA, Newbold D, Reding ME, rasheed A, Blodgett J, Kornman KS. The relationship between reduction in periodontal inflammation and diabetes control: a report of 9 cases. J Periodontol 1992; 63: 83848

18. Aldridge JP, Lester V, Watts TLP, Collins A, Viberti G, Wilson RF. Single-blind studies of the effects of improved periodontal health on metabolic control in Type-I diabetes mellitus. J Clin Periodontol 1995; 22: 271-275

19. Grossi SG, Skrepcinski FB, DeCaro T, Zambon JJ, Cummins D, Genco RJ. Response to periodontal therapy in diabetics and smokers. J Periodontol 1996; 67: 1094-1102

20. Brownlee M. Glycation and diabetic complications. Diabetes 1994; 43: 836-841

21. Offenbacher S. Periodontal diseases: pathogenesis. Ann Periodontal 1996; 1:821-978

22. Papapanou P. Periodontal diseases: Epidemiology. Ann Periodontol 1996; 1: 1-37

23. Löe H. Periodontal disease. The sixth complication of diabetes mellitus. Diabetes Care 1993; 16: 329-334

24. Nathan DM. Long-term complications of diabetes mellitus. N Engl J Med 1993; 328: 1676-1685

25. Rayfeld EJ, Ault MJ, Keush GT, Brothers MJ, Nechemias C, Smith $\mathrm{H}$. Infection and diabetes: The case for glucose control. Am J Med 1982; 72: 439-450

26. Ling PR, Bistrian BR, Mendez B, Istfan NW. Effects of systemic infusions of endotoxin, tumor necrosis factor, and interleukin-1 on glucose metabolism in the rat: Relationship to endogenous glucose production and peripheral tissue glucose uptake. Metab Clin Exp 1994; 43: $279-284$

27. Lonroth P. Regulation of insulin action at the cellular level. J Inter Med 1991; 73 (suppl.): 23-29

28. Kanety H, Feinstein R, Papa MZ, Hemi R, Karasik A. Tumor necrosis factor alpha-induced phosphorylation of insuline receptor substrate-1 (IRS-1). Possible mechanism of suppression of insulin-stimulated tyrosine phosphorylation of IRS-1. J Biol Chem 1995; 270: 23780-23784 
29. Clark CM, Lee DA. Prevention and treatment of the complications of diabetes. N Engl J Med 1995; 332: 1210-1217

30. Socransky SS, Haffajee AD. Microbial mechanisms in the pathogenesis of destructive periodontal diseases: a critical assessment. J Periodont Res 1991; 26: 195-212

31. Haffajee A, Socransky SS. The etiologic agents of destructive periodontal disease. Periodontol 2000. 1994; 5 : 78-111

32. Darveu RP, Tanner A, Page R. The microbial challenge in periodontitis. Periodontol 2000. 1997; 14: 12-32

33. Dennison DK, Van Dyke TE. The acute inflammatory response and the role of phagocytic cells in periodonal health and diseasse. Periodontol 2000. 1997; 14: 54-78

34. Ishikawa I, Nakashima $\mathrm{K}$, Koseki T, et al. Induction of the immune response to periodontopathic bacteria and its role in the pathogenesis of periodontitis. Periodontol 2000. 1997; 14: 79-111

35. Brownlee M. Clycation products and the pathogenesis of diabetic complications. Diabetes Care 1992; 15: 18351843

36. Brownlee M. Glycation and diabetic complications. Diabetes 1994; 43: 836-841

37. Schmidt AM, Weidman E, Lalla E, et al. Advanced glycation end products (AGES) induce oxidant stress in the gingiva: A potential mechanism underlying accelerated periodontal disease associated with diabetes. J Periodont Res 1996; 31: 508-515

38. Kornman KS, Page RC, Tonetti MS. The host response to the microbial challenge in periodontitis: Assembling the player. Periodontol 2000. 1997; 14: 33-53

39. Nishimura F, Takahashi K, Kurihara M, Takashiba S, Murayama Y. Periodontal disease as a complication of diabetes mellitus. Ann Periodontol 1998; 3 (1): 20-29

40. Monouchehr-Pour M, Spagnuolo HM, Bissada NF. Impaired neutrophil chemotaxis in diabetic patients with severe periodontitis. J Dent Res 1981; 60: 729-730

41. Bissada NF, Monouchehr-Pour M, Haddow M, Spagnuolo PJ. Neutrophil functional activity in juvenile and adult onset diabetic patients with mild and severe periodontitis. J Periodont Res 1982; 17: 500-502

42. Nishimura F, Terranova VP, Foo $H$, Kurihara M, Kurihara $H$, Murayama Y. Glucose-mediated alteration of celular function in human periodontal ligament cells. J Dent Res 1996; 75: 1664-1671
43. Manouchehr-Pour M, Spagnuolo PJ, Rodman HM, Bissada NF. Comparison of neutrophil chemotactic response in diabetic patients with mild and severe periodontal disease. J Periodontol 1981; 52: 410-415

44. Iacono VJ, Singh S, Golub LM, Ramamurthy NS, Kaslick R. In vivo assay of crevicular leukocyte migration. Its development and potential applications. J Periodontol 1985; 56 (suppl. 2): 56-62

45. Manouchehr-Pour M, Spagnuolo PJ, Bissada NF. Impaired neutrophil chemotaxis in diabetic patients with severe periodontitis. J Dent Res 1981; 60: 729-730

46. McMullen JA, Van Dyke TE, Horoszewicz HU, Genco RJ. Neutrophil chemotaxis in individuals with advanced periodontal disease and a genetic predisposition to diabetes mellitus. J Periodontol 1981; 52: 167-173

47. Bissada NF, Manouchehr-Pour M, Haddow M, Spagnuolo PJ. Neutrophil functional activity in juvenile and adult onset diabetic patients with mild and severe periodontitis. J Periodontol Res 1982; 17: 500-502

48. Golub LM, Nicoll GA, Iacono VJ, Ramamurthy NS. In vivo crevicular leucocyte response to a chemotactic challenge: Inhibition by experimental diabetes. Infect immune 1982; 37: 1013-1020

49. Cutler CW, Eke P, Arnold RR, van Dyke TE. Defective neutrophy function in on insulin-dependent diabetes mellitus patient. J Periodontol 1991; 62: 394-401

50. Willershauschen-Zonchen B, Lemmen C, Hamm G. Influence of high glucose concentrations on glycosaminoglycan and collagen synthesis in cultured human gingival fibroblasts. J Clin Periodontol 1991; 18: 190-195

51. Sorsa T, Ingman T, Soumalainen $\mathrm{K}$, et al. Cellular source and tetracycline inhibition of gingival crevicular fluid collagenase of patients with labile diabetes mellitus. J Clin Periodontol 1992; 19: 146-149

52. Baynes J. Role of oxidative stress in development of complications in diabetes. Diabetes 1991; 40: 405-412

53. Neeper M, Schmidt AM, Brett J, et al. Cloning and expression of RAGE: A cell surface receptor for AGEs. J Biol Chem 1992; 267: 14998-15004

54. Schneir ML, Ramamurthy NS, Golub LM. Extensive degradation of recently synthesized collagen in gingival of normal and streptozotocin-induced diabetic rats. J Dent Res 1984; 63: 23-27 
